\title{
Combining RMC and PDF methods to characterize amorphous drug
}

\author{
Vinicius D. N. Bezzon ${ }^{1}$, João C. de Lima², Fabio F. Ferreira ${ }^{1}$ \\ ${ }^{1}$ Federal University of ABC, Center for Natural and Human Sciences, Santo André - SP, Brazil \\ ${ }^{2}$ Federal University of Santa Catarina, Florianópolis - SC, Brazil
}

vinicius.bezzon@gmail.com

Drugs in a crystalline state are preferably used due to their physicochemical stability [1]. Around $70 \%$ of the drug candidates to be newly available drugs have low solubility, which can compromise bioavailability and, consequently, product development [2]. Therefore, amorphous phases have become more attractive to the industry field as a promising strategy to improve solubility. The amorphous structure can be defined as a long-range tridimensional molecular packing loss. Compared to its crystalline counterpart, the amorphous can have higher inner energy that can increase the drug solubility, dissolution rate, and extension, improving the final formulation bioavailability. Several processes can be used to reach the drug's amorphous structure, such as solvent evaporation, melting-cooling, lyophilization, and ball-milling. However, the amorphous drug metastability can induce structure recrystallization, which can be a problem [3]. Furthermore, different amorphization processes can promote local molecular packing variations yielding changes in the properties.

Herein, we combined the pair distribution function (PDF) and Reverse Monte Carlo (RMC) methods with data from a high-resolution diffractometer (model STADI-P, STOE ${ }^{\circledR}$ ) equipped with a MoK $\alpha_{1}$ source, available at the "Laboratory of Crystallography and Structural Characterization of Materials" (LCCEM) at the Federal University of ABC, Santo André-SP, Brazil to analyze crystalline drugs under amorphization process. By the results, we could identify the amorphization mechanisms when using ball-mill and solvent evaporation process for different drugs, which can be induced by displacing $\mathrm{O}-\mathrm{O}$ and $\mathrm{O}-\mathrm{H}$ correlations (molecule distortions) as well as variations in hydrogen bonds. In both cases, the molecule was preserved after the amorphization process.

[1] D.A. Snider, W. Addicks, W. Owens, Polymorphism in generic drug product development. (2004). Adv. Drug Deliv. Rev. 56 $391-395$.

[2] B.Y. Shekunov, P. York, Crystallization processes in pharmaceutical technology and drug delivery design. (2000). J. Cryst. Growth. 211, 122136.

[3] S.L. Raghavan, A. Trividic, A.F. Davis, J. Hadgraft, Crystallization of hydrocortisone acetate: influence of polymers. (2001). Int. J. Pharm. 212, 213-221.

Keywords: pair distribution function; Reverse Monte Carlo; amorphous drug.

The authors thank the financial support of The São Paulo Research Foundation (FAPESP) grant \#2018/11990-5 and the National Council for Scientific and Technological Development (CNPq) grant \#305661/2019-9. 